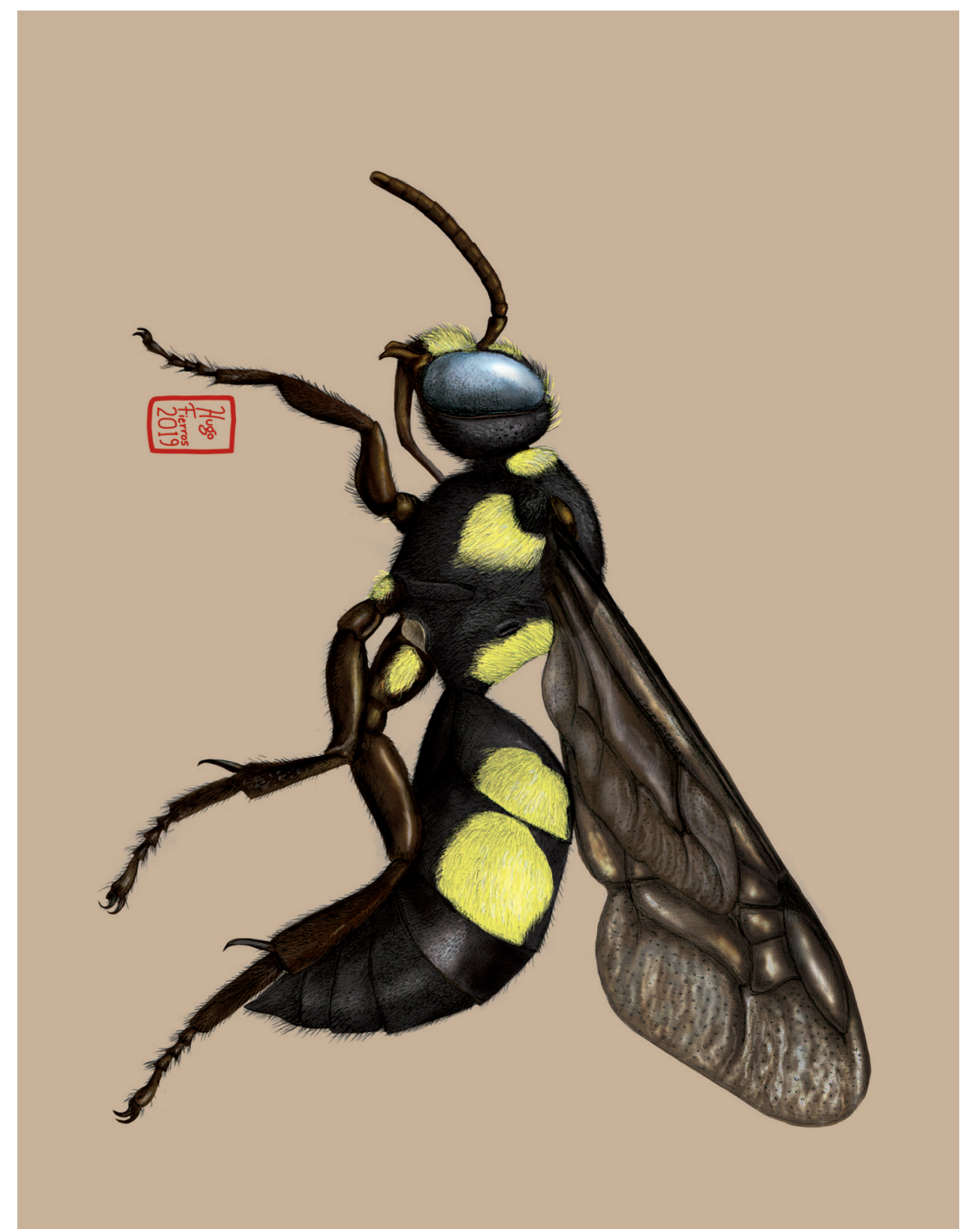

Dugesiana, Año 27, No. 1, enero 2020-junio 2020 (primer semestre de 2020), es una publicación semestral, editada por la Universidad de Guadalajara, a través del Centro de Estudios en Zoología, por el Centro Universitario de Ciencias Biológicas y Agropecuarias. Camino Ramón Padilla Sánchez \# 2100, Nextipac, Zapopan, Jalisco, Tel. 37771150 ext. 33218, http://148.202.248.171/dugesiana/index.php/DUG/index, glenusmx@gmail.com. Editor responsable: José Luis Navarrete-Heredia. Reserva de Derechos al Uso Exclusivo 04-2009-062310115100-203, ISSN: 2007-9133, otorgados por el Instituto Nacional del Derecho de Autor. Responsable de la última actualización de este número: José Luis Navarrete-Heredia, Editor y Ana Laura González-Hernández, Asistente Editorial. Fecha de la última modificación 1 de enero 2020, con un tiraje de un ejemplar.

Las opiniones expresadas por los autores no necesariamente reflejan la postura del editor de la publicación.

Queda estrictamente prohibida la reproducción total o parcial de los contenidos e imágenes de la publicación sin previa autorización de la Universidad de Guadalajara. 


\title{
Dendroctonus (Coleoptera: Curculionidae) y su relación con la temperatura en San Cristóbal de Las Casas, Chiapas
}

\author{
Dendroctonus (Coleoptera: Curculionidae) and its relationship with temperature in San Cristóbal de Las \\ Casas, Chiapas
}

Carlos Eduardo Aguilar-Castillo ${ }^{1 *}$, Eduardo Aguilar-Astudillo² y Alicia Niño Dominguez ${ }^{3}$

${ }^{1}$ Coordinación de la Licenciatura en Caficultura, Universidad Autónoma de Chiapas. ${ }^{2}$ Facultad de Ciencias Agronómicas, Campus V, Universidad Autónoma de Chiapas. El Colegio de la Frontera Sur (ECOSUR). ${ }^{~}$ Carretera Jaltenango a Francisco I. Madero Km. 2.6 s/n, C.P. 30370, Ángel Albino Corzo, Chiapas, México. ${ }^{2}$ Carretera Ocozocoautla - Villaflores km. 84.5. Apartado postal \#78, C.P. 30470, Villaflores, Chiapas, México. ${ }^{3}$ Carretera Antiguo Aeropuerto km 2.5, C.P. 30700, Tapachula, Chiapas, México. *Autor de correspondencia: carlos_casti1 (a)outlook.com

\section{RESUMEN}

Se determinó la fluctuación poblacional de escarabajos descortezadores de pino del género Dendroctonus, en el municipio de San Cristobal de las Casas, Chiapas, México. La investigación se realizo durante un periodo anual que comprendió de noviembre de 2016 a noviembre de 2017, Se determinaron dos sitios de muestreo, usando trampas multiembudo tipo Lindgren cebadas con semioquímicos. Los datos se sometieron a un análisis descriptivo realizando graficas de fluctuación poblacional por especie y género, para determinar la relación entre la temperatura (mínima, media y máxima) y la fluctuación poblacional por especie del género Dendroctonus Erichson, 1836, se aplicó el análisis de correlación de Pearson. Se capturaron en total 1,981 ejemplares del género Dendroctonus, distribuidas en seis especies: D. frontalis Zimmermann, 1868, D. adjunctus Blandford, 1897, D. approximatus Dietz, 1890, D. valens LeConte, 1860, D. parallelocollis Chapuis, 1869 y D. mesoamericanus Armendáriz-Toledano y Sullivan, 2015, las mayores capturas se registraron durante la temporada calurosa en las estaciones de primavera y verano, con excepción de $D$. frontalis que se capturó en mayor abundancia durante la temporada fría en el mes de diciembre de 2016. La relación entre la temperatura y la variación poblacional es mejor correlacionada con la temperatura máxima, en comparación con la temperatura mínima y media, además, la población del género Dendroctonus presento mayor abundancia en el período caluroso con temperaturas entre los 24 y $28.8^{\circ} \mathrm{C}$, en las estaciones de primavera y verano.

Palabras clave: Descortezadores, Fluctuación poblacional, D. frontalis, D. adjunctus.

\begin{abstract}
The population fluctuation of pine bark beetles of the genus Dendroctonus, in the municipality of San Cristobal de Las Casas, Chiapas, Mexico, was determined. The investigation was carried out during an annual period from November 2016 to November 2017. Two sampling sites were determined, using Lindgren multi-funnel traps primed with semiochemicals. The data were subjected to a descriptive analysis by making population fluctuation graphs by species and genus, to determine the relationship between temperature (minimum, medium and maximum) and population fluctuation by species of the genus Dendroctonus Erichson, 1836, the analysis of Pearson's correlation. A total of 1,981 specimens of the genus Dendroctonus were captured, distributed in six species: D. frontalis Zimmermann, 1868, D. adjunctus Blandford, 1897, D. approximatus Dietz, 1890, D. valens LeConte, 1860, D. parallelocollis Chapuis, 1869 and D. mesoamericanus Armendáriz-Toledano and Sullivan, 2015, the highest catches were recorded during the hot season in the spring and summer seasons, with the exception of $D$. frontalis which was captured in greater abundance during the cold season in the month of December 2016. The relationship between temperature and population variation is better correlated with the maximum temperature, in comparison with the minimum and average temperature, in addition, the population of the genus Dendroctonus presented greater abundance in the hot period with temperatures between 24 and $28.8^{\circ} \mathrm{C}$, in the spring and summer seasons.
\end{abstract}

Keywords: Bark beetles, Population fluctuation, D. frontalis, D. adjunctus.

Los escarabajos del género Dendroctonus Erichson, 1836 (Coleoptera: Curculionidae: Scolytinae), conocidos comúnmente como descortezadores de pino (SalinasMoreno et al. 2010), son insectos con 20 especies descritas a nivel mundial, 18 se encuentran en América y dos en Europa y Asia (Wood 1982; Armendáriz-Toledano y Zúñiga 2017). México presenta la mayor diversidad de Dendroctonus con 13 especies registradas, de ellas, las especies $D$. mexicanus
Hopkins, 1905, D. frontalis Zimmermann, 1868, D. adjunctus Blandford, 1897, D. approximatus Dietz, 1890, D. valens LeConte, 1860, D. parallelocollis Chapuis, 1869 y D. mesoamericanus Armendáriz-Toledano y Sullivan, 2015, están reportadas para el estado de Chiapas (Zúñiga et al. 1999; Salinas-Moreno et al. 2010; ArmendárizToledano et al. 2015). 
Los descortezadores son importantes componentes de los bosques de pino al contribuir con la regeneración y restauración del bosque al matar arboles enfermos, dañados o debilitados por factores naturales o antropogénicos (Six y Bracewell 2015), mientras que el incremento de sus poblaciones a niveles epidémicos, los convierte en un importante factor de disturbio en función de la temperatura que modifica la distribución y densidad poblacional, incrementando los daños e impacto en los bosques de pino y como consecuencia provoca desequilibrio ecológico y pérdidas económicas en la industria forestal (Raffa y Berryman 1987; FAO 2010; CONAFOR 2013).

En México, la mayor parte de los trabajos sobre fluctuación poblacional del género Dendroctonus se han realizado en el norte y centro de la republica mexicana, por los problemas de infestación y las grandes extensiones de bosques de estas zonas (Morales-Rangel et al. 2016; Mendoza-Villa y Obregón-Zúñiga 2016). Mientras que en los estados del sureste como Guerrero, Oaxaca y Chiapas, los estudios de estos organismos son muy escasos (Altúzar 2010; Domínguez-Sánchez 2010; Leal 2014), a pesar de ser reportados como uno de los principales factores de disturbio (Castellanos et al. 2013; Macías-Sámano et al. 2004; Niño et al. 2015; Niño et al. 2016). Las investigaciones de Domínguez-Sánchez et al. (2008) y Altúzar (2010) proporcionan información sobre la fluctuación poblacional de descortezadores, al mencionar que $D$. frontalis es abundante en los meses de julio a diciembre, lo cual difiere con la población de las especies $D$. frontalis y $D$. mexicanus reportadas para el estado de Hidalgo al presentarse en mayor abundancia, esto se sabe por las investigaciones realizadas por Avilés-Carrillo et al, (2016) y Hernández-Muñoz y Obregón-Zúñiga (2016), para ambos estudios se capturaron más de 8000 individuos en los meses de marzo a junio, descendiendo drásticamente en los meses restantes (julio a diciembre) con menos de 2000 individuos capturados,

Con base en lo expuesto anteriormente, se realizó el presente trabajo de investigación para conocer la fluctuación poblacional de las especies del género Dendroctonus y su relación con la temperatura durante el periodo de diciembre 2016 a noviembre de 2017 en el municipio de San Cristobal de Las Casas, Chiapas.

\section{Zonas de estudio}

\section{MATERIALES Y MÉTODO}

La investigación se realizó del 19 de noviembre de 2016 al 30 de noviembre de 2017, en dos sitios del municipio de San Cristobal de Las Casas, Chiapas, el primero ubicado dentro de las instalaciones de El Colegio de la Frontera Sur (ECOSUR) en las coordenadas $16^{\circ} 66^{\prime} 97^{\prime \prime} \mathrm{N}, 92^{\circ} 55^{\prime}$ $88^{\circ} \mathrm{O}$, con $2350 \mathrm{msnm}$ de elevación y el segundo ubicado dentro de las instalaciones del Instituto Nacional de Investigaciones Forestales Agrícolas y Pecuarias (INIFAP) en las coordenadas $16^{\circ} 44^{\prime} 12^{\prime \prime} \mathrm{N}, 92^{\circ} 38^{\prime} 18^{\prime \prime} \mathrm{O}$, con 2180 msnm de elevación. El clima de ambos sitios de estudio, según el sistema de clasificación de Koppen modificado por García (1973), es templado subhúmedo con lluvias abundantes en verano (C (w2)). La temperatura media anual varía desde los 12 a $24^{\circ} \mathrm{C}$, la precipitación total alcanza los 1500 mm (INEGI, 2010; INEGI, 2012).

\section{Establecimiento de trampas y colecta de muestras}

En cada sitio de muestreo se colocaron seis trampas multiembudo de tipo Lindgren de 8 unidades, las trampas se colocaron dentro de una hectárea de área, cada una separada $50 \mathrm{~m}$ entre sí abarcando $300 \mathrm{~m}$, el establecimiento de trampas y el cebado con tratamientos semioquímicos se realizo según la literatura de Macías y Niño (2016): T1= alfa/beta-pineno + frontalina + endo-Brevicomina, $\mathrm{T} 2=\mathrm{alfa} /$ beta-pineno + frontalina (testigo) y, T3= alfa/beta-pineno + frontalina + endo-brevicomina + ipsdienol, respetando la dosis establecida por Synergy Semiochemicals Corporation para cada feromona (frontalina, endo-Brevicomina e ipsdianol) y kairomona (alfa/beta-pineno) empleada en la investigación. La designación de los tratamientos se realizó bajo el diseño experimental de bloques aleatorizados con dos réplicas en cada sitio. Los cebos fueron reemplazados cada dos meses considerando las condiciones climáticas del municipio. El vaso colector fue provisto de $200 \mathrm{ml} \mathrm{de}$ etilenglicol (anticongelante) para preservar las muestras capturadas, renovando cada 15 días el anticongelante.

Los datos de temperatura (mínima, media y máxima) fueron proporcionados por la Comisión Nacional del Agua (CONAGUA) de la Estación Sinóptica Meteorológica de San Cristóbal de las Casas, Chiapas; ubicada en las coordenadas $16^{\circ} 43^{\prime} 28.87^{\prime \prime} \mathrm{N}, 92^{\circ} 38^{\prime} 13.83^{\prime \prime} \mathrm{O}$, ubicada a 1 $\mathrm{km}$ de distancia a la zona de estudio.

Las muestras se recogieron cada 15 días y se guardaron en bolsas tipo Ziploc $\AA$, los cuales se trasladaron al laboratorio de Sanidad Forestal en El Colegio de la Frontera Sur (ECOSUR) unidad Tapachula y a la Universidad de Ciencias y Artes de Chiapas (UNICACH) en Tuxtla Gutiérrez, Chiapas, en donde se separaron e identificaron los escarabajos del género Dendroctonus mediante el uso de claves taxonómicas dicotómicas de Wood (1982), Armendáriz-Toledano et al. (2015), Armendáriz-Toledano y Zúñiga (2017). Los descortezadores identificados se separaron y se contabilizaron por especie y fecha de muestreo.

\section{Análisis de datos}

Con los datos del conteo de individuos de cada especie, se realizaron análisis de forma quincenal y estacional, durante el año de muestreo (primavera, verano, otoño e invierno), determinando los meses y estaciones de mayor y menor abundancia de descortezadores. Para determinar el grado de relación entre la temperatura (mínima, media y máxima) y la abundancia de cada especie de descortezador se realizó un análisis de correlación de Pearson con el programa PAleontological STatistics (PAST3) versión 3.20. 


\section{RESULTADOS}

Se colectaron 1,981 individuos del género Dendroctonus cuya identificación corresponde a seis especies: $D$. adjunctus, $D$. frontalis, $D$. approximatus, $D$. mesoamericanus, D. valens y D. parallelocollis (Cuadro 1).

De manera general, la población del género Dendroctonus fluctuó de manera constante durante el ciclo anual de muestreo, encontrando mayor número de individuos capturados en el mes de mayo y en la estación de primavera. Las especies $D$. adjunctus y $D$. frontalis se encontraron en mayor abundancia con 47.50 y $28.37 \%$, mientras que la especie $D$. parallelocollis fue la de menor abundancia con $0.25 \%$, del total de especies capturados del género Dendroctonus.

Se determino que cada especie de Dendroctonus, presenta momentos particulares de mayor y menor incremento poblacional, las mayores abundancias se registraron durante la temporada calurosa que abarcan las estaciones de primavera y verano, mientras que las menores abundancias en la temporada fría en las estaciones de otoño e invierno, esto principalmente por el aumento y la disminución de la temperatura durante el ciclo anual de monitoreo (Cuadro 2).

El análisis de Pearson determinó que el género Dendroctonus presenta correlación con la temperatura máxima al presentar valores significativos de $\mathrm{r}^{2}=0.61 \mathrm{y}$ $p=0.040$, mientras que el análisis de forma estacional indica que hay correlación con las estaciones de invierno con la temperatura mínima y primavera con la temperatura máxima al presentar valores de $\mathrm{r}^{2}=0.794, \mathrm{p}=0.044 \mathrm{y} \mathrm{r}^{2}=$ $0.610, p=0.040$ respectivamente, dichos valores indican que conforme la temperatura aumente, la población de los escarabajos descortezadores tienden a crecer o a disminuir drásticamente, modificando su ciclo biológico, aumentando su periodo de vuelo e infestación.

El análisis de correlación de Pearson de forma anual para cada especie indica que $D$. mesoamericanus y $D$. approximatus, presentan correlación con la temperatura máxima $\left(26.1\right.$ a $\left.28.8{ }^{\circ} \mathrm{C}\right)$ y media $\left(17.5\right.$ a $\left.18.5{ }^{\circ} \mathrm{C}\right)$, mientras que $D$. frontalis se correlaciona únicamente con la temperatura máxima $\left(18.3\right.$ a $\left.28.8{ }^{\circ} \mathrm{C}\right)$, al presentar

Cuadro 1. Especies del género Dendroctonus identificadas y porcentaje de captura.

\begin{tabular}{|c|c|c|}
\hline \multirow{2}{*}{ Especie } & \multicolumn{2}{|c|}{ Captura } \\
\hline & Total & $\%$ \\
\hline D. adjunctus & 941 & 47.50 \\
\hline D. frontalis & 562 & 28.37 \\
\hline D. approximatus & 171 & 8.64 \\
\hline D. mesoamericanus & 163 & 8.22 \\
\hline D. valens & 139 & 7.01 \\
\hline D. parallelocollis & 5 & 0.26 \\
\hline Total & 1981 & 100 \\
\hline
\end{tabular}

valores de $\mathrm{r}^{2}=0.161 \mathrm{y} \mathrm{p}=0.038$ (Cuadro 3 ). Por otra parte, el análisis de correlación de forma estacional, determinó que las especies $D$. frontalis y $D$. valens se correlacionan significativamente con la temperatura máxima, la primera de ellas en primavera con temperaturas entre los 24.9 a $28.8^{\circ} \mathrm{C}$, al presentar valores de $\mathrm{r}^{2}=0.795 \mathrm{y} \mathrm{p}=0.012$ y la segunda en invierno con temperaturas entre los 24 a 26.1 ${ }^{\circ} \mathrm{C}$, con valores de $\mathrm{r}^{2}=0.823$ y $\mathrm{p}=0.025$ respectivamente, la diferencia radica en la estación de inicio de aumento poblacional para cada especie, pero son similares, al aumentar considerablemente sus poblaciones si se presenta un cambio mínimo en la temperatura, acelerando su reproducción y distribución por el ambiente, provocando mayores afectaciones en los bosques de pino.

\section{DISCUSIÓN}

Los descortezadores del género Dendroctonus muestran una distribución restringida por la fluctuación de la temperatura, de las 13 especies de escarabajos distribuidas en México, solo D. pseudotsugae, D. rhizophagus, $D$. brevicomis, D. mexicanus, D. approximatus, $D$. mesoamericanus, $D$. adjunctus, $D$. valens, $D$. frontalis y $D$. parallelocollis muestran mejor adaptación y desarrollo a elevaciones entre los 2500 y $4000 \mathrm{~m}$, en donde se presenta variación climática muy marcada en las estaciones conforme al cambio drástico en las condiciones de precipitación, temperatura y humedad ambiental (Armendáriz-Toledano y Zúñiga 2017), por el cual, en el municipio de San Cristóbal de Las Casas, las condiciones de temperatura permiten que los descortezadores $D$. approximatus, D. mesoamericanus, $D$. adjunctus, $D$. valens, $D$. frontalis y $D$. parallelocollis se distribuyan y cumplan con sus funciones biológicas y ecológicas de forma exitosa, pero una variación en la temperatura ambiental provoca que estos organismos proliferen excesivamente hasta ser consideradas plagas (Zúñiga et al. 1999; Salinas-Moreno et al. 2010).

La abundancia diferencial para cada una de las especies, sugiere que $D$. adjunctus es el escarabajo descortezador mejor adaptado a las condiciones climáticas del municipio, al ser la especie de mayor abundancia poblacional, esta información concuerda con resultados reportados por Salinas-Moreno et al. (2010) y Armendáriz-Toledano y Zúñiga (2017), al mencionar que $D$. adjunctus está mejor adaptada a temperaturas por debajo de $\operatorname{los} 28{ }^{\circ} \mathrm{C} \mathrm{y}$ altitudes desde los 2100 a $3000 \mathrm{msnm}$. En cambio, para los descortezadores $D$. frontalis y $D$. mesoamericanus, los datos arrojan que bajo estas condiciones altitudinales no es el mejor sitio de desarrollo para ambas especies, principalmente por las condiciones ambientales que se presentan en estas altitudes (temperatura, precipitación, humedad, entre otros.), autores como Moreno et al. (2008) y Niño et al. (2016), presentan mayores capturas en altitudes entre los 1500 a $1700 \mathrm{msnm}$, con temperaturas entre los 16 a $22{ }^{\circ} \mathrm{C}$. Para el resto de los descortezadores, la abundancia en bajas proporciones (menos de 500 individuos capturados) sugiere que los tratamientos 
Cuadro 2. Mes y estación del año de mayor y menor abundancia poblacional de las especies del género Dendroctonus.

\begin{tabular}{lcccc}
\hline \multirow{2}{*}{ Especie } & \multicolumn{4}{c}{ Abundancia } \\
\cline { 2 - 5 } & Diciembre & Noviembre & Primavera & Otoño \\
\hline \multirow{2}{*}{ D. frontalis } & $(167)$ & $(14)$ & $(172)$ & $(40)$ \\
& Abril & Noviembre & Primavera & Otoño \\
D. adjunctus & $(131)$ & $(4)$ & $(492)$ & $(42)$ \\
& Mayo & Noviembre & Primavera & Otoño \\
D. approximatus & $(49)$ & $(5)$ & $(103)$ & $(10)$ \\
& Junio & Noviembre & Primavera & Otoño \\
D. mesoamericanus & $(30)$ & $(6)$ & $(67)$ & $(16)$ \\
& Julio & Noviembre & Primavera & Otoño \\
D. valens & $(22)$ & $(6)$ & $(67)$ & $(12)$ \\
D. parallelocollis & Mayo, Julio & Septiembre & Verano & Primavera \\
\hline
\end{tabular}

Cuadro 3. Correlación de Pearson para la abundancia poblacional de cada especie del género Dendroctonus en un ciclo anual y la variación de la temperatura mínima, media y máxima.

\begin{tabular}{|c|c|c|c|c|}
\hline \multirow{2}{*}{ Especie } & \multirow{2}{*}{ Correlación } & \multicolumn{3}{|c|}{ Temperatura ${ }^{\circ} \mathrm{C}$} \\
\hline & & Mínima & Media & Máxima \\
\hline \multirow{2}{*}{ D. adjunctus } & $r^{2}$ & 0.033 & 0.016 & 0.132 \\
\hline & $p$ & 0.372 & 0.532 & 0.065 \\
\hline \multirow{2}{*}{ D. frontalis } & $\mathrm{r}^{2}$ & -0.028 & 0.002 & 0.161 \\
\hline & $p$ & 0.413 & 0.831 & 0.038 \\
\hline \multirow{2}{*}{ D. mesoamericanus } & $r^{2}$ & 0.079 & 0.163 & 0.160 \\
\hline & $p$ & 0.170 & 0.033 & 0.043 \\
\hline \multirow{2}{*}{ D. approximatus } & $r^{2}$ & 0.003 & 0.168 & 0.339 \\
\hline & $p$ & 0.800 & 0.016 & 0.001 \\
\hline \multirow{2}{*}{ D. valens } & $r^{2}$ & 0.032 & 0.093 & 0.023 \\
\hline & $p$ & 0.384 & 0.125 & 0.462 \\
\hline
\end{tabular}

semioquímicos utilizados no son los adecuados para su captura, probablemente porque las mezclas utilizadas son empleadas para la atracción de especies primarias y no secundarias como estos descortezadores, Niño-Domínguez et al. (2016) y Macías y Niño (2016), sugieren que para la captura de estas especies de descortezadores se utilizan tratamientos compuestos principalmente por frontalina (feromona) + alfa/beta-pineno (kairomona), el cual ayuda a la atracción de descortezadores secundarios, pero impide la atracción de descortezadores primarios como $D$. adjunctus.

Los descortezadores adaptados a condiciones de altitudes mayores, tales como $D$. pseudotsugae, D. rhizophagus, $D$. mexicanus, D. adjunctus, D. brevicomis, D. approximatus, D. mesoamericanus, D. valens y $D$. parallelocollis, distribuidos principalmente en regiones altas en diversos estados de la republica mexicana (Chihuahua, Durango, Querétaro, Guerrero, entre otros) en altitudes entre los 2500 a $4000 \mathrm{msnm}$ presentan diferencias en su desarrollo como ciclo biológico más cortos con pocas generaciones al año e inclusive llegan a ser univoltinas (Armendáriz-Toledano y Zúñiga 2017), en contraste, para el estado de Chiapas, principalmente para el municipio de San Cristobal de Las 
Casas, las especies D. adjunctus, D. approximatus, D. mesoamericanus, D. valens y D. parallelocollis, se ubican en altitudes entre los 2180 a 2350 msnm, en estas altitudes es común que estos descortezadores aumenten su abundancia poblacional y colonicen nuevos hospederos (dañados o estresados por factores naturales o antropogénicos) para cumplir de manera eficiente con sus ciclos biológicos, autores como Hansen y Betz (2003), Pérez-De la Cruz et al. (2009) y Leal (2014), mencionan que temperaturas mayores a los $29^{\circ} \mathrm{C}$ en estaciones que abarcan la temporada calurosa (primavera y verano), provoca que los descortezadores alcancen su máximo desarrollo e inician su etapa de vuelo y dispersión, sincronizándola con las estaciones e inicio de estrés fisiológico y susceptibilidad de sus hospederos, provocando incremento en los eventos de infestación por el aumento poblacional de los descortezadores, provocando pérdidas económicas y ecológicas en los bosques de pino.

El conteo de especies del género Dendroctonus por meses y estaciones del año, determino que cada descortezador presenta momentos característicos de mayor y menor abundancia poblacional. El descortezador $D$. adjunctus fue abundante durante la temporada calurosa en la estación de primavera en los meses de abril y mayo del 2017, en cambio las menores abundancias se registraron en los meses de julio y septiembre del 2017. Estos resultados concuerdan con los obtenidos por Rodríguez (2009) y Rodríguez et al. (2013), pero difieren con los meses de menor captura, ya que las menores abundancias de $D$. adjunctus se dan durante la temporada fría en los meses de octubre a diciembre. El trabajo realizado por RodríguezOrtega et al. (2010), menciona que $D$. adjunctus los meses de mayor abundancia poblacional se presentan en marzo y septiembre con 81 y 124 individuos capturados, dichos resultados difieren con esta investigación, al encontrar que septiembre es el mes de menor abundancia poblacional con menos de cuatro individuos colectados. Es probable que la variación en la captura de descortezadores en cada uno de los trabajos se deba a la diferencia en las condiciones ambientales, principalmente de temperatura, así como de las condiciones latitudinales y altitudinales de cada una de las áreas de estudio. Por otra parte, la población del descortezador $D$. frontalis fluctuó de manera constante durante el ciclo anual de monitoreo (diciembre 2016 a noviembre 2017), sin sobrepasar los 60 ejemplares capturados, presentando mayor incremento poblacional en el mes de diciembre de 2016 (otoño e invierno) con 167 individuos y mayo de 2017 (primavera) con 97 individuos capturados respectivamente. De acuerdo a Macías (2001) y Altúzar (2010), la alta abundancia poblacional de $D$. frontalis en diciembre corresponde a su ciclo biológico natural, en que los adultos emergen para formar una nueva generación, pero de acuerdo a Lombandero et al. (2000) y Morales-Rangel et al. (2016), estos resultados son atípicos, ya que estos organismos son poiquilotérmicos y por lo consiguiente, sí se presenta una disminución en la temperatura la población tiende a decrecer, en cambio si la temperatura aumenta considerablemente la población de D. frontalis incrementa provocando grandes infestaciones en los bosques de pino. Tal vez, las altas poblaciones de D. frontalis durante la temporada fría, en San Cristobal de las Casas, se deba porque no intervienen otros factores climáticos, tales como humedad relativa, precipitación, viento, entre otros, que eviten su proliferación, emergencia y vuelo, así como la propagación de feromonas (frontalina y endo-brevicomina) que sirven para la atracción de estos descortezadores, Cuellar-Rodríguez et al. (2012) y Leal (2014), mencionan que otros factores como la precipitación o humedad relativa pueden afectar la densidad de los insectos o que debido a la misma se pierda el efecto atrayente de las feromonas, provocando una disminución drástica en la abundancia poblacional, o en cambio, al combinar las variables precipitación, humedad relativa y temperatura se pueden obtener mejores resultados en correlación con el número de insectos colectados en trampas, debido a que los semioquímicos se liberan y dispersan mejor por las condiciones ambientales, por ejemplo: los semioquímicos se volatilizan fácilmente cuando las condiciones ambientales son cálidas y secas, generando mayor atracción de insectos hacia las trampas, mientras que en periodos fríos y húmedos las feromonas no alcanzan niveles atractivos para los insectos.

En comparación con las especies D. adjunctus y D. frontalis, los descortezadores $D$. approximatus, $D$. mesoamericanus, $D$. valens y $D$. parallelocollis, presentan su mayor abundancia poblacional en la temporada calurosa en los meses de abril a julio, en las estaciones de primavera y verano, Pérez-De la Cruz et al. (2009), mencionan que durante los meses de marzo a septiembre, en las estaciones de primavera y verano, las temperaturas oscilan entre los 24 y $29{ }^{\circ} \mathrm{C}$, los descortezadores presentan su mayor actividad biológica y de vuelo, puesto que en ellas se encuentran las mejores condiciones para colonizar nuevos hospederos e iniciar nuevamente su ciclo biológico, como en el caso del descortezador $D$. valens que durante al final de la temporada fría (febrero) e inicio de la temporada calurosa (marzo) se observa un incremento en su densidad poblacional pasando de cero individuos colectados hasta llegar su mayor abundancia poblacional en verano, específicamente en julio con más de 22 ejemplares capturados.

Como organismos poiquilotermos o exotermos, para los descortezadores de pino el desarrollo de su progenie, así como también su vuelo y dispersión, son afectados por la variación de la temperatura. Bentz (2009) y Leal (2014), mencionan que un aumento en la temperatura, entre los $16{ }^{\circ} \mathrm{C}$ y $18{ }^{\circ} \mathrm{C}$ en adelante, provoca que en las diferentes estaciones del año se produzca un aceleramiento en el índice de desarrollo de los escarabajos del género Dendroctonus, así como un aumento en su capacidad reproductiva, principalmente para los descortezadores $D$. adjunctus y $D$. frontalis. Lo anterior es corroborado con lo reportado en este trabajo al encontrar mayor abundancia poblacional en las estaciones de primavera y verano coincidiendo con el 
incremento de la temperatura, cuyo valor promedio varia dentro del rango de los $14.2{ }^{\circ} \mathrm{C}-18.6{ }^{\circ} \mathrm{C}$ y $16.9{ }^{\circ} \mathrm{C}-17.5$ ${ }^{\circ} \mathrm{C}$ para cada una de las especies del género Dendroctonus presentes en el municipio de San Cristobal de las Casas, Chiapas. Estos datos sugieren que la temperatura media por debajo de los $16.8{ }^{\circ} \mathrm{C}$ afecta considerablemente la dispersión de los descortezadores del género Dendroctonus de esta investigación. Por el contrario, la temperatura media por arriba de los $17.5^{\circ} \mathrm{C}$ incrementa drásticamente el desarrollo y abundancia de los descortezadores reflejándose en un periodo de vuelo mayor, ya que para todas las especies del género Dendroctonus ( $D$. frontalis, D. adjunctus, $D$. mesoamericanus, $D$. approximatus, $D$, valens y $D$. parallelocollis), las mayores abundancias de las capturas coincide con un incremento de la temperatura media entre $\operatorname{los} 17.5^{\circ} \mathrm{C}$ y $18.3^{\circ} \mathrm{C}$.

Comparado con los resultados generales, el incremento de la temperatura máxima en la estación de primavera fue el periodo con los mejores valores de correlación entre esta variable y la abundancia poblacional de los descortezadores $D$. approximatus, $D$. mesoamericanus y $D$. frontalis. Esta última especie, además, presentó correlación con la temperatura máxima en primavera cuando su abundancia poblacional fue mayor, mientras que para la especie $D$. valens la correlación fue mejor explicada en la época de invierno.

Es probable que para $D$. frontalis y $D$. valens el desarrollo de su progenie, dependa fuertemente de las condiciones fisiológicas de los arboles de pino (hospederos), debido a que el incremento de la temperatura particularmente entre los 16 y $18{ }^{\circ} \mathrm{C}$ en adelante, en los arboles de pino se induce un periodo de estrés hídrico, mientras que en los escarabajos descortezadores se inicia un incremento en su población, modificando drásticamente su periodo de vuelo y dispersión, si bien ésta es una condición que favorece la probabilidad del éxito de colonización de estos insectos, el grado de estrés hídrico en sus hospederos puede afectar el desarrollo óptimo de la progenie de los descortezadores y su abundancia (Macías y Niño 2016).

Resultados similares han sido reportados para los estados de Hidalgo y Querétaro, por trabajos realizados por Avilés-Carrillo et al. (2016), Hernández-Muñoz y Obregón-Zúñiga (2016), Morales-Rangel et al. (2016) y Mendoza-Villa y Obregón-Zúñiga (2016), todos ellos con base a la baja correlación entre la fluctuación poblacional de descortezadores del género Dendroctonus y la temperatura, principalmente para las especies $D$. frontalis y $D$. adjunctus, al presentar picos de mayor abundancia poblacional en una sola estación del año o dos, mientras que para el resto de la investigación la población disminuye drásticamente sin que la temperatura descienda de igual manera, entonces se dice que mientras una variable aumenta (temperatura o abundancia de individuos) la otra disminuye y viceversa, afectando drásticamente los arboles de pino o la abundancia de los escarabajos descortezadores del género Dendroctonus. Algunos de estos trabajos indican que otras variables como la precipitación o la humedad relativa pueden explicar mejor esta relación, al afectar de forma positiva o negativa la población de los descortezadores al aumentar o disminuir conforme a las variables climáticas.

\section{AGRADECIMIENTOS}

Al USDA-FS- Southern Station por otorgar el recurso económico para realizar el estudio. Al Dr. Pablo Liedo responsable del proyecto de colaboración entre ECOSURTAP- USDA-FS. por apoyar el desarrollo del proyecto en ECOSUR unidad Tapachula. Al Dr. Brian Sullivan por su asesoría y apoyo en la realización de esta investigación. Al M.C. Benigno Gómez y Gómez por su participación como asesor y dar las facilidades de operación en el laboratorio de ECOSUR. Al Biol. Juan Antonio López y al Ing. Gabriel Domínguez por su apoyo técnico en laboratorio y campo.

\section{LITERATURA CITADA}

Altúzar, M.R. 2010. Implementación de un sistema de trampeo para el monitoreo de Dendroctonus frontalis en el Parque Nacional Lagunas de Montebello, Chiapas, México. Tesis de Licenciatura. Universidad Autónoma Agraria "Antonio Narro". Coahuila, México.

Armendáriz-Toledano, F. and G. Zúñiga. 2017. Illustrated key to species of genus Dendroctonus (Coleoptera: Curculionidae) occurring in Mexico and Central America. Journal of Insect Science, 17(2): 34; 1-15.

Armendáriz-Toledano, F., A. Niño, B.T. Sullivan, L.R. Kirkendall and G. Zúñiga. 2015. A New Species of Bark Beetle, Dendroctonus mesoamericanus sp. nov. (Curculionidae: Scolytinae), in Southern Mexico and Central America. Annals of the Entomological Society of America, 108 (3): 403-414.

Avilés-Carrillo, I., S. Vergara-Pineda, V.H. CambrónSandoval y J.A. Obregón-Zúñiga. 2016. Fluctuación poblacional de Dendroctonus frontalis Zimmermann, 1868 y Dendroctonus mexicanus Hopkins, 1909 (Curculionidae: Scolytinae) en relación a la variación en la altitud y factores climáticos en un bosque de pino en Zimapán, Hidalgo. Entomología Mexicana, 3: 649655.

Castellanos, B. J. F., M.E.O. Ruiz, C.M. Gómez y C.R. González. 2013. Guía metodológica para combatir plagas de descortezadores en el sur de México. Instituto Nacional de Investigaciones Forestales Agrícolas y Pecuarias (INIFAP). Centro Regional Pacífico Sur. Oaxaca, México.

Comisión Nacional Forestal (CONAFOR). 2013. Bosques, cambio climático y REDD+ en México, guía básica. Jalisco, México.

Cuéllar-Rodríguez, G., A. Equihua-Martínez, E. EstradaVenegas, T. Méndez-Montiel, J. Villa-Castillo y J. Romero-Nápoles. 2012. Fluctuación poblacional de Dendroctonus mexicanus Hopkins (Coleoptera: Curculionidae: Scolytinae) atraídos a trampas en el noreste de México y su correlación con variables 
climáticas. Boletín del Museo de Entomología de la Universidad del Valle, 13 (2): 12-19.

Domínguez-Sánchez, B., J. Macías-Sámano, N. RamírezMarcial y J.J. León-Cortés. 2008. Respuesta kairomonal de coleópteros asociados a Dendroctonus frontalis y dos especies de Ips (Coleoptera: Curculionidae) en bosques de Chiapas, México. Revista Mexicana de Biodiversidad, 79: 176-183.

Hansen, M.E. and B.J. Bentz. 2003. Comparison of reproductive capacity among univoltine, semivoltine, and re-emerged parent spruce beetles (Coleoptera: Scolytidae). The Canadian Entomologist, 135(5): 697712.

Hernández-Muñoz， G. y J. Obregón-Zúñiga. 2016. Fluctuación poblacional de descortezadores (Coleoptera: Curculionida: Scolytinae) en bosques de pino (Pinus sp.) en Zimapán, Los Mármoles, Tlaxco, Hidalgo, México. Entomología mexicana, 3: 639-643.

Instituto Nacional de Estadística Geográfica e Información (INEGI). 2010. Compendio de información geográfica municipal 2010; San Cristobal de Las Casas, Chiapas. Secretaría de Programación y Presupuesto del Estado de Chiapas. Chiapas, México.

Instituto Nacional de Estadística Geográfica e Información (INEGI). 2012. México en Cifras. Información nacional, por entidad federativa y municipios. San Cristóbal de Las Casas, Chiapas. http://www3.inegi.org.mx/ sistemas/mexicocifras/default.aspx?e $=7$. Consultado el día 25 de octubre de 2019.

Leal, O.N. 2014. Fluctuación poblacional de Dendroctonus mexicanus Hopkins y variación estacional de la temperatura y humedad relativa, en San Juan del Estado, ETLA, Oaxaca. Tesis de Maestría. Colegio de Postgraduados. Edo. de México, México.

Lombandero, J.M., P.M. Ayres, D.B. Ayres and J.D. Reeve. 2000. Cold tolerance of four species of bark beetle (Coleoptera: Scolytidae) in North America. Environmental Entomology, 29 (3): 421-432.

Macías, J.E. 2001. Mediación semioquímica entre insectos descortezadores y árboles de coníferas. (pp. 450-483). En: Anaya, A.L., Espinosa-García, F. y R.R. CruzOrtega.Relaciones químicas entre organismos aspectos básicos y perspectivas de su aplicación. Plaza y Valdez editores. México, D.F.

Macías-Sámano. J.E., A. Niño-Domínguez., J.A. CruzLópez y R. Altúzar-Mérida. 2004. Monitoreo de descortezadores y sus depredadores mediante el uso de semioquímicos: Manual operativo. ECOSURCONAFOR-CONANP-USDA FOREST SERVICE. Tapachula, Chiapas, México.

Macías, S.J.E. y A. Niño. 2016. Protocolo para monitoreo de descortezadores de coníferas mediante el uso de atrayentes y semioquímicos. Imprenta Caleb. Chiapas, México.

Mendoza-Villa, O.N. y J.A. Obregón-Zúñiga. 2016. Cambio en la abundancia de Dendroctonus frontalis
Zimmerman, 1868 y Dendroctonus mexicanus Hopkins, 1909 (Coleoptera: Curculionidae: Scolytinae) en un gradiente altitudinal en el cerro "La Pingüica", Pinal de Amoles, Querétaro. Entomología Mexicana, 3: 644648.

Moreno, B., J. Macías, B. Sullivan and R.R. Clarke. 2008. Field response of Dendroctonus frontalis (Coleoptera: Scolytinae) to synthetic semiochemicals in Chiapas, México. Journal of Economic Entomology, 101(6): 1821-1825.

Morales-Rangel, A., V.H. Cambrón-Sandoval, S. VergaraPineda y A. Obregón-Zúñiga. 2016. Fluctuación poblacional de Dendroctonus frontalis Zimmerman, 1868 y Dendroctonus mexicanus Hopkins, 1909 (Coleoptera: Curculionidae: Scolytinae) y su asociación con variables climáticas en bosques de pino en el municipio de Landa de Matamoros, Querétaro, México. Entomología Mexicana, 3: 633-638.

Niño, A., S.T. Sullivan, J. López-Urbina and MacíasSámano, J.E. 2015. Pheromone-mediated mate location and discrimination by two syntopic sibling species of Dendroctonus bark beetles in Chiapas, Mexico. Journal of Chemical Ecology, (41):746-756.

Niño-Domínguez, A., B.T. Sullivan, J.H. López-Urbina and J.E. Macías-Sámano. 2016. Responses by Dendroctonus frontalis and Dendroctonus mesoamericanus (Coleoptera: Curculionidae) to semiochemical Lures in Chiapas, Mexico: Possible Roles of Pheromones During Joint Host Attacks. Journal of Economic Entomology, 109(2): 724-731.

Nordhaus, H. and M. Stephenson. 2005. Bark beetles and temperature. (pp. 10-12). In: Bentz, B.J. (Eds.). Bark beetle outbreaks in western North America: causes and consequences. Bark Beetle Symposium. Snowbird, Utah, USA.

Organización de las Naciones Unidas para la Alimentación y la Agricultura (FAO). 2010. Evaluación de los recursos forestales mundiales 2010, informe principal. Estudio FAO: Montes 163. Roma, Italia.

Pérez-De la Cruz, M., A. Equihua-Martínez, J. RomeroNápoles, S. Sánchez-Soto y E. García-López. 2009. Diversidad, fluctuación y plantas huésped de escolitinos (Coleoptera: Curculionidae) asociados con el agroecosistema cacao en Tabasco, México. Revista Mexicana de Biodiversidad, (80): 779-791.

Rodríguez, O.A. 2009. Fluctuación de Dendroctonus adjunctus Blandford y sus depredadores atraídos por frontalina + alfa-pineno, en Los Pescados, Veracruz y en Zoquiapan, Estado de México. Tesis de Doctorado. Colegio de Postgraduados. Edo. de México, México.

Rodríguez-Ortega, A., A. Equihua-Martínez, J. CibriánTovar y E.G. Estrada-Venegas. 2010. Fluctuación de Dendroctonus adjunctus Blandford (Curculionidae: Scolytinae) y sus depredadores atraídos por frontalina+alfa-pineno, en la estación experimental de Zoquiapan, Edo. de México. Boletín del Museo de 
Entomología de la Universidad del Valle, 11 (1): 20-27. Rodríguez, O.A., M.A.Equihua, T.J.Cibrián, V.E.G.Estrada, M.J.T. Méndez, C.J. Villa, y Y.R.M. Barrón. 2013. Fluctuación de Dendroctonus adjuntus (Coleoptera: Curculionidae: Scolytidae) y sus depredadores atraídos por frontalina + alfa-pineno, en los Pescados, Veracruz, México. Revista Chilena de Entomología, (38): 41-50.

Raffa, F.K. and A.A. Berryman. 1987. Interacting selective pressures in conifer-bark beetle systems: a basis for reciprocal adaptations. The American Naturalist, 2(129): 234-262.

Salinas-Moreno, Y., A. Ager, C.F. Vargas, J.L. Hayes, and G. Zúñiga. 2010. Determining the vulnerability of Mexican pine forests to bark beetles of the genus Dendroctonus Erichson (Coleoptera: Curculionidae: Scolytinae). Forest Ecology and Management, (250): 52-61.

Recibido: 11 de julio 2019

Aceptado: 5 de noviembre 2019
Salinas-Moreno, Y., M.C.F. Vargas, G. Zúñiga, J. Victor, A. Ager y J.L. Hayes. 2010. Atlas de distribución geográfica de los descortezadores del género Dendroctonus (Curculionidae: Scolytinae) en México. Instituto Politécnico Nacional-Comisión Nacional Forestal. México.

Six, D.L. and R. Bracewell. 2015. Dendroctonus. (pp. 305339). In: Vega, F. and R. Hofstetter (Eds.). Biology and ecology of native and invasive species. Academic Press, Elsevier Inc. UK, London.

Wood, S.L. 1982. The bark and ambrosia beetles of North and Central America (Coleóptera: Scolytidae), A taxonomic monograph. Great Basin Natural Memories, (6): 1-1359.

Zúñiga, G., C.G. Mendoza, R. Cisneros y Y. SalinasMoreno. 1999. Zonas de sobreposición en las áreas de distribución geográfica de las especies mexicanas de Dendroctonus Erichson (Coleoptera: Scolytidae) y sus implicaciones Ecológico-evolutivas. Acta Zoológica Mexicana, 77: 1-22. 